\title{
Analysis of Individual Case Safety Reports of Severe Cutaneous Adverse Reactions in Korea
}

\author{
Min-Gyu Kang ${ }^{1,2,3}$, Kyung-Hee Sohn ${ }^{1,4}$, Dong-Yoon Kang, ${ }^{5,6}$, Han-Ki Park ${ }^{1,7}$, \\ Min-Suk Yang ${ }^{1,8}$, Ju-Yeun Lee ${ }^{9}$, and Hye-Ryun Kang ${ }^{1,5,6,10}$ \\ ${ }^{1}$ Institute of Allergy and Clinical Immunology, Seoul National University Medical Research Center, Seoul; \\ ${ }^{2}$ Department of Internal Medicine, Chungbuk National University Hospital, Cheongju; \\ ${ }^{3}$ Regional Pharmacovigilance Center, Chungbuk National University Hospital, Cheongju; \\ ${ }^{4}$ Department of Internal Medicine, Kyung-Hee University Hospital, Seoul; \\ ${ }^{5}$ Regional Pharmacovigilance Center, Seoul National University Hospital, Seoul; \\ ${ }^{6}$ Drug Safety Monitoring Center, Seoul National University Hospital, Seoul; \\ ${ }^{7}$ Department of Internal Medicine, School of Medicine, Kyungpook National University, Kyungpook National University Chilgok Hospital, Daegu; \\ ${ }^{8}$ Department of Internal Medicine, Seoul Metropolitan Government-Seoul National University Boramae Medical Center, Seoul; \\ ${ }^{9}$ College of Pharmacy, Seoul National University, Seoul; \\ ${ }^{10}$ Division of Allergy and Clinical Immunology, Department of Internal Medicine, Seoul National University College of Medicine, Seoul, Korea.
}

\begin{abstract}
Purpose: Despite morbidities and fatalities, nationwide epidemiologic data for severe cutaneous adverse reactions (SCARs), including Stevens-Johnson syndrome (SJS), toxic epidermal necrolysis (TEN), and drug reaction with eosinophilia and systemic symptoms (DRESS), are not widely available. We aimed to investigate SCAR epidemiology over the last two decades in Korea.

Materials and Methods: We analyzed individual case safety reports (ICSRs) of SCARs in the Korea Adverse Event Reporting System from 1988 to 2013. Administered drugs, demographic profiles, and causality assessment according to the World Health Organization-Uppsala Monitoring Center system were analyzed.

Results: A total of 755 SCAR cases (508 SJS/TEN, 247 DRESS) were reported. The number of SCAR ICSRs has been increasing with increasing ICSRs for overall adverse drug events. Since 2010, the number of SCAR ICSRs has increased up to 100 cases/year. Allopurinol was the most common causative drug (SJS/TEN: 10.2\%; DRESS: 11.3\%; SCAR ICSRs: 10.6\%), followed by carbamazepine (SJS/TEN: 8.7\%; DRESS: 9.7\%; SCAR ICSRs: 8.6\%). Regarding drug groups, antiepileptics (19.5\%) and antibiotics for systemic use $(12.7 \%)$ were common causative drug groups. Twenty SCAR-related deaths were recorded. Antibacterials were the most common causes of deaths ( 8 cases), followed by antiepileptics ( 5 cases). The potential risk of SCARs was not specified in the drug information leaflet for $40.2 \%$ of drugs causing SJS/TEN and $82.5 \%$ causing DRESS syndrome in Korea.

Conclusion: The number of SCAR ICSRs has increased rapidly with recent active pharmacovigilance programs in Korea. Allopurinol and antiepileptics are the most common individual and categorical causative agents, respectively.
\end{abstract}

Key Words: Pharmacovigilance, Stevens-Johnson syndrome, toxic epidermal necrolysis, drug hypersensitivity syndrome

Received: September 19, 2018 Revised: December 21, 2018

Accepted: December 29, 2018

Corresponding author: Hye-Ryun Kang, MD, PhD, Department of Internal Medicine, Seoul National University Hospital, 101 Daehak-ro, Jongno-gu, Seoul 03080, Korea.

Tel: 82-2-2072-0820, Fax: 82-2-742-3291, E-mail: helenmed@snu.ac.kr

-The authors have no potential conflicts of interest to disclose.

(C) Copyright: Yonsei University College of Medicine 2019

This is an Open Access article distributed under the terms of the Creative Commons Attribution Non-Commercial License (https://creativecommons.org/licenses/ by-nc/4.0) which permits unrestricted non-commercial use, distribution, and reproduction in any medium, provided the original work is properly cited.

\section{INTRODUCTION}

An adverse drug reaction (ADR) is defined as an unintended harmful reaction to a drug. ${ }^{1}$ With advances in medicine, people are taking more medications, and adverse drug events (ADEs) are rapidly increasing. Lazzarou, et al. ${ }^{2}$ reported that ADRs are the fourth leading cause of death in the US, after heart disease, cancer, and stroke. According to the Global Burden of Disease report of 2013, the number of worldwide ADR-related deaths was 142000 in 2013, a 1.5-fold increase over that reported in 
1990. ${ }^{3}$ Thus, ADR is an important medical issue related to patient safety and a heavy socioeconomic burden.

Cutaneous ADR is the most common type of ADR and can be caused by many widely used medications. ${ }^{4}$ Despite cutaneous ADRs being usually self-limited, some ADEs can manifest as severe cutaneous adverse reactions (SCARs), such as Stevens-Johnson syndrome (SJS), toxic epidermal necrolysis (TEN), and drug reactions with eosinophilia and systemic symptoms (DRESS) syndrome. Although early diagnosis of SCARs is crucial, it is difficult to differentiate SCARs at initial presentation from other disease conditions with cutaneous manifestations. Therefore, the mortality due to SCARs is considerably high (20-25\%), and morbidities causing lasting disabilities are also relatively common. ${ }^{5}$

The epidemiology of SCARs seems to be different between countries. SCAR development is known to be significantly influenced by both genetic background and the pattern of drug usage. Previous studies have reported that the epidemiology of SCARs in Korean people seem different from that in Europeans, as well as the Han Chinese in East Asia. ${ }^{6,7}$ However, to date, there has been no nationwide epidemiologic study of SCARs in Korea.

In this study, we aimed to investigate the epidemiology and common causative drugs of SCARs by reviewing individual case safety reports (ICSRs) of the Korea Adverse Event Reporting System (KAERS) since its introduction in 1988.

\section{MATERIALS AND METHODS}

\section{KAERS}

The KAERS is an ADE reporting system developed by the Korea Institute of Drug Safety and Risk Management (KIDS), an official agency for managing drug safety. It contains ICSRs of any suspected ADEs voluntarily reported by healthcare personnel, consumers, and manufacturers. Each ICSR contains the following information: 1) demographic profile of the patient with the suspected ADE, 2) phenotype coded according to World Health Organization Adverse Reactions Terminology (WHOART 092 version), seriousness, and outcome, 3) causative drug or drugs and/or co-administered drugs coded according to the Anatomical Therapeutic Chemical (ATC) classification system and the dose/route/frequency/duration of these medications, and 4) causality assessment performed by specialists of each regional pharmacovigilance center according to the WHOUppsala Monitoring Center (UMC) criteria.

\section{Selection of SCAR ICSRs}

This study was approved by the Review Board of Korea Institute of Drug Safety and Risk Management. We extracted ICSRs from 1988 to 2013 that were in line with WHO-ART codes from the KIDS-KAERS database (KIDS-KD): 1) SJS, WHO-ART code “0042/001/0014/0100"; 2) TEN, WHO-ART code
“0013/005/0014/0100"; and 3) DRESS syndrome, WHO-ART code "2309/002/1810/." ICSRs of drug hypersensitivity syndrome (WHO-ART code “2309/001/1810/"), which was formerly usually used to describe DRESS-like symptoms, were also extracted. Two allergy specialists with experience in pharmacovigilance independently reviewed the detailed information in each ICSR of suspected SCARs and rechecked the causality assessment and culprit drugs (Fig. 1). Among the ICSRs of suspected SCARs extracted from the KIDS-KD, only the ICSRs in which two allergy specialists assessed the causality as "possible," "probable," or "certain" were included for analysis. Some ICSRs coded for drug hypersensitivity syndrome were re-categorized into ICSRs for DRESS syndrome if they matched the diagnostic criteria for DRESS syndrome. For the assessment of causative drugs, we included ICSRs in which a single drug was listed as the causative drug or in which multiple drugs were listed but co-administration was usually regarded as standard therapy, such as anti-tuberculosis treatments. We did not consider ICSRs of SCARs in which all the co-administered drugs were listed as causative drugs or there was no detailed information to assess the culprit drug based on the history of ICSRs. To minimize the possibility of duplication or overestimation of SCAR ICSRs, reports from pharmaceutical companies based on literature reviews or consumer protection agencies lacking drug information or causality assessments were excluded from analysis.

\section{RESULTS}

\section{Summary of SCAR ICSRs}

From 1988 to 2013, a total of 478519 ADEs were spontaneously included in the KAERS. Of those, 755 cases $(0.16 \%)$ were of SCARs, comprising 508 cases of SJS/TEN (67.3\%) and 247 cases of DRESS syndrome (32.7\%). Since the reporting of the first SCAR case in 1991, an increasing tendency has been observed for SCAR ICSRs (Fig. 2). Since 2010, more than 100 SCAR ICSRs have been submitted annually. Despite the increasing tendency of SCAR ICSRs, the proportion of SCAR ICSRs among all ICSRs did not increase $(0.19 \%$ in $2010,0.25 \%$ in $2011,0.17 \%$ in 2012, and $0.11 \%$ in 2013).

The clinical characteristics of SCAR ICSRs are described in Table 1 . The mean age of patients with SCARs was $50.3 \pm 20.7$ years (mean $\pm \mathrm{SD}$ ), of which $28.4 \%$ were older adults (age: 65 years or more) and $8.2 \%$ were children/adolescents (age: 18 years or less). Male patients accounted for $48.7 \%$ of the total ICSRs. The mean age and proportion of the older adult patients with SJS/TEN were not significantly different from those with DRESS syndrome (53.1 \pm 17.8 years vs. $48.8 \pm 22.0$ years and $29.7 \%$ vs. $27.7 \%$, respectively). Further, the proportion of childhood/ adolescent patients was significantly higher in the SJS/TEN group than in the DRESS group ( $11.1 \%$ vs. $2.6 \%$, respectively, $p<0.01)$. 


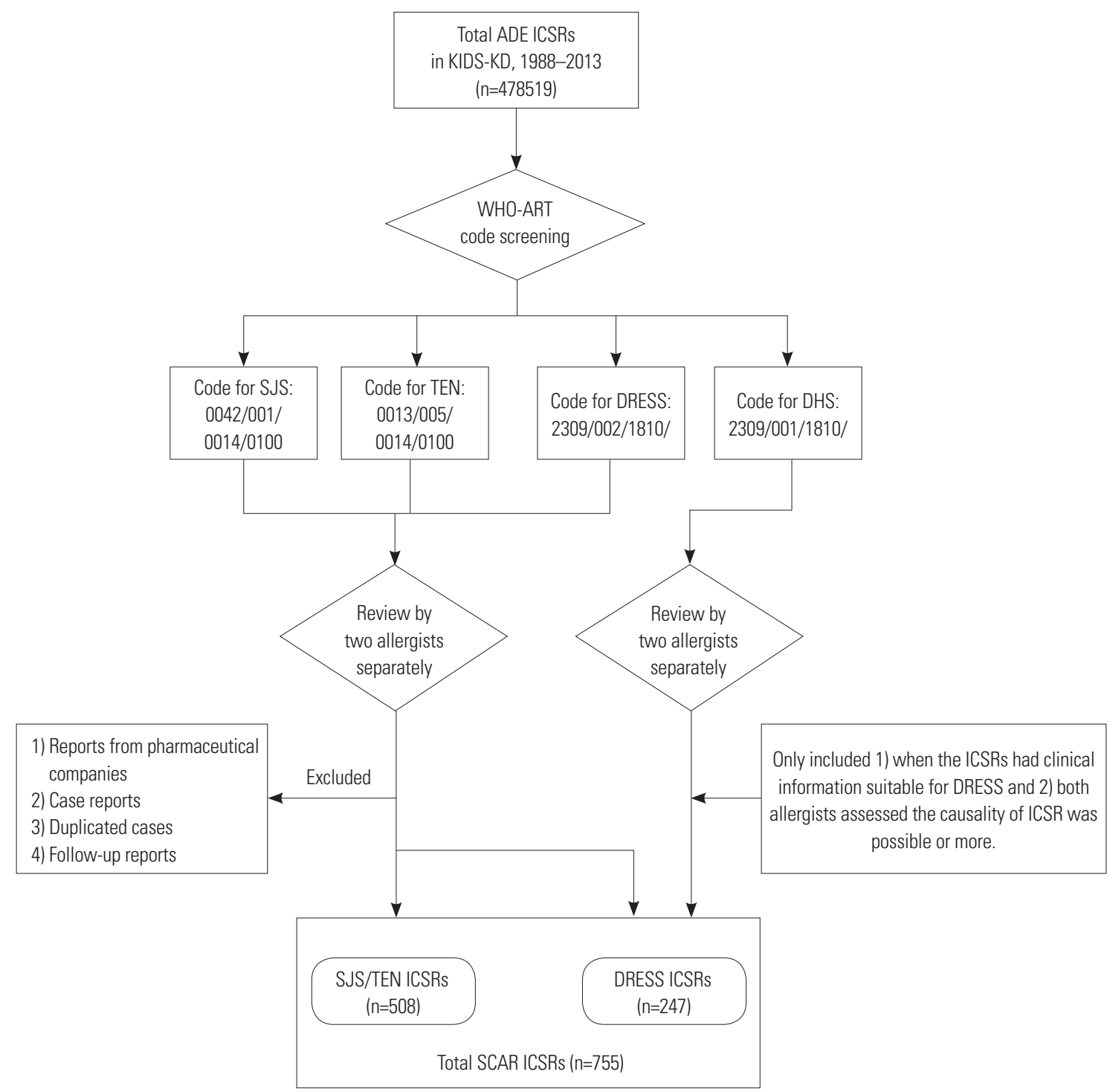

Fig. 1. Scheme of the selection process for ICSRs of SCARs in the KIDS-KD. ICSRs, individual case safety reports; SCARs, severe cutaneous adverse reactions; KIDS-KD, Korea Institute of Drug Safety and Risk Management-Korea Adverse Event Reporting System database; ADE, adverse drug event; SJS, Stevens-Johnson syndrome; TEN, toxic epidermal necrolysis; DRESS syndrome, drug reaction with eosinophilia and systemic symptoms syndrome; DHS, drug hypersensitivity syndrome; WHO-ART, World Health Organization Adverse Reactions Terminology.

The most common drug administration route was oral (72.3\%), followed by intravenous injection (25.8\%), subcutaneous injection (1.0\%), and eye-drop instillation (0.9\%). The drug administration route was not different between the DRESS and SJS/TEN groups ( $p>0.05)$. The median time for symptom latency (from drug administration to symptom onset) was 19 days, with a mean $\pm S D$ of $24.6 \pm 21.5$ days. The median time for symptom latency was longer in the DRESS group than in the SJS/TEN group, by a median of 11 days ( $31.2 \pm 26.0$ days vs. $20.4 \pm 17.0$ days, $p<0.05)$.

\section{Causative drugs}

The most common causative drug in the SCAR ICSRs was allopurinol (10.6\%), the proportion of which was the highest in both the DRESS and SJS/TEN groups (Table 2), followed by carbamazepine (8.6\%), lamotrigine (4.4\%), methazolamide (2.1\%), valproic acid $(2.1 \%)$, vancomycin (1.6\%), dapsone (1.5\%), paracetamol (1.3\%), amoxicillin and/or enzyme inhibitor (1.2\%), piperacillin and/or enzyme inhibitor (1.2\%), and sulfamethoxazole and trimethoprim (1.2\%). Table 2 shows detailed information for causative drugs listed in SCAR ICSRs.

According to the ATC subgroups, antiepileptics (ATC code: N03A) were the most common causative drugs ( $19.5 \%$ of total SCAR ICSRs), followed by antibacterials (ATC code: J01A, $12.7 \%$ ), anti-gout preparations (ATC code: M04A, 10.6\%), and non-steroidal anti-inflammatory drugs (NSAIDs, ATC code: M01A, 4.7\%).

Among antiepileptics, carboxamide derivatives (ATC code N03AF, 9.7\% of total SCAR ICSRs) including carbamazepine and oxcarbazepine were the most common SCAR-causing 


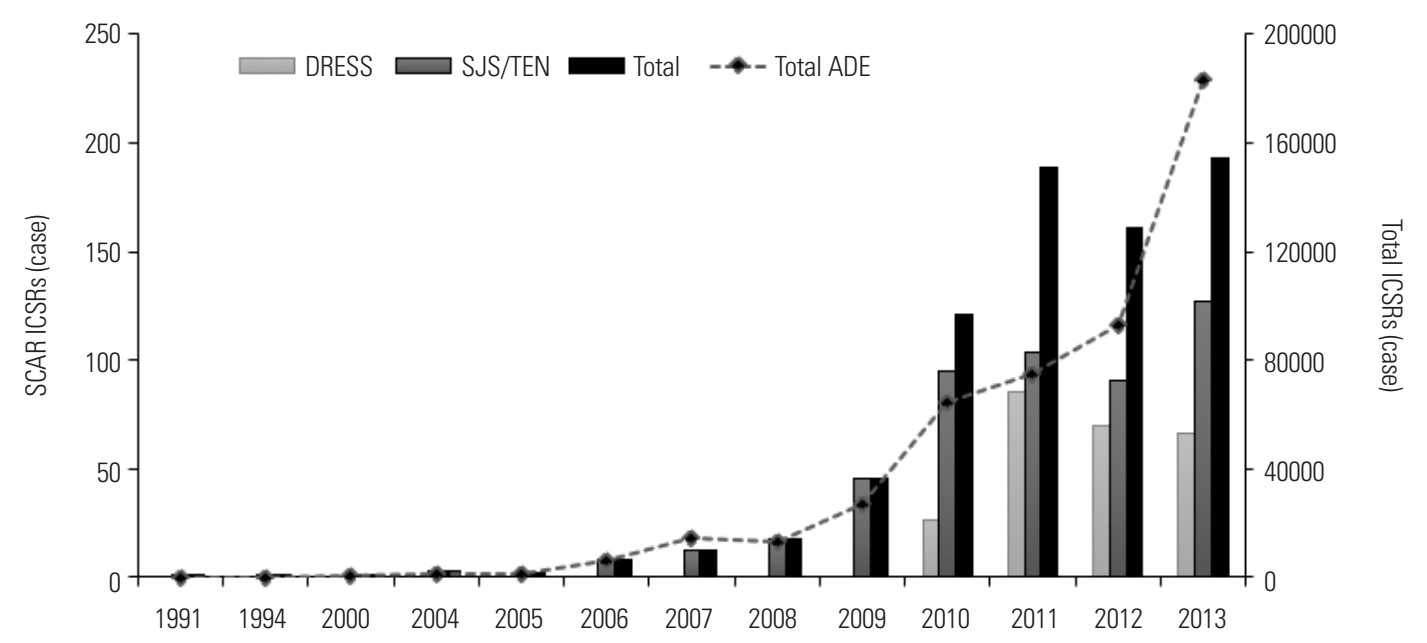

Fig. 2. Trends in annual numbers of ICSRs of SCARs in KAERS. ICSRs, individual case safety reports; SCARS, severe cutaneous adverse reactions; KAERS, Korea Adverse Event Reporting System; SJS, Stevens-Johnson syndrome; TEN, toxic epidermal necrolysis; DRESS syndrome, drug reaction with eosinophilia and systemic symptoms syndrome.

Table 1. Baseline Characteristics in Individual Case Safety Reports of SCARs

\begin{tabular}{|c|c|c|c|}
\hline & Total & DRESS syndrome & SJS/TEN \\
\hline Number of self-reports & 755 & 247 & 508 \\
\hline Age (mean $\pm S D, y r)$ & $50.3 \pm 20.7$ & $53.1 \pm 17.8$ & $48.8 \pm 22.0$ \\
\hline Elderly ( $\geq 65$ yrs) (\%) & 28.4 & 29.7 & 27.7 \\
\hline Children/adolescents ( $\leq 18$ yrs) (\%) & 8.2 & 2.6 & 11.1 \\
\hline Male sex (\%) & 48.7 & 49.2 & 48.5 \\
\hline Number of causative drugs (mean $\pm S D$ ) & $1.6 \pm 1.2$ & $1.5 \pm 0.9$ & $1.6 \pm 1.2$ \\
\hline Latent period [median (mean \pm SD), days] & $19(24.6 \pm 21.5)$ & $27(31.2 \pm 26.0)$ & $16(20.4 \pm 17.0)$ \\
\hline \multicolumn{4}{|l|}{ Route of administration (\%) } \\
\hline Oral & 72.3 & 70.6 & 72.8 \\
\hline Intravenous injection & 25.8 & 27.5 & 25.3 \\
\hline Others (subcutaneous or eye-drop instillation) & 1.9 & 1.8 & 2.0 \\
\hline
\end{tabular}

SD, standard deviation; SCARs, severe cutaneous adverse reactions; DRESS syndrome, drug reaction with eosinophilia and systemic symptoms syndrome; SJS, Stevens-Johnson syndrome; TEN, toxic epidermal necrolysis.

drugs, followed by other antiepileptics including lamotrigine (ATC code N03AX, 6.2\%), fatty acid derivatives including valproic acid (ATC code: N03AG, 2.1\%), and hydantoin derivatives including phenytoin (ATC code: N03AB, 1.1\%). Among antibacterials, cephalosporin (ATC code: J01DB, J01DC, J01DD, and J01DE) was the most common SCAR-causing antibacterial (3.6\% of total SCAR ICSRs), followed by beta-lactam antibacterials; penicillins (ATC code: J01C, $2.8 \%$ ) including ampicillin, amoxicillin, and piperacillin; other antibacterials including glycopeptide antibacterials (ATC code: J01X, 1.7\%); quinolone antibacterials (ATC code: J01M, 1.5\%); and sulfonamides and trimethoprim (ATC code: J01E, 1.2\%). Among NSAIDs (ATC code: M01A, 4.4\%), propionic acid derivatives (ATC code: $\mathrm{M} 01 \mathrm{AE}$ ) including ibuprofen and dexibuprofen were the most common SCAR-causing NSAIDs (2.0\%), followed by acetic acid derivatives and related substances including diclofenac or aceclofenac (ATC code: M01AB, 0.8\%), coxibs (ATC code: M01AH, 0.5\%), and fenamates (ATC code: M01AG, 0.5\%). Fig. 3 shows the detailed proportions of SCAR-causing drugs ac- cording to the pharmacologic main group (level 2) or pharmacologic/therapeutic subgroup (level 3) per the WHO's ATC classification system.

\section{Drug labeling information}

We reviewed the drug labeling information provided by the Korea Pharmaceutical Information Center (http://www.health. $\mathrm{kr}$ ) to examine whether the potential to cause SCARs was specified in the drug labeling information (Table 2). The labeling information of $59.8 \%$ of the 61 SJS/TEN-causing drugs specified the potential risk for SJS/TEN. In almost all SJS/TEN cases (90.2\%), SJS/TEN was caused by drugs whose potential to cause the condition was specified in the drug labeling information. With regard to DRESS, the labeling information of only 14 of $80(17.5 \%)$ causative drugs specified the potential risk for DRESS. Approximately $60 \%$ of DRESS cases were attributable to drugs whose labeling information did not specify the potential to cause DRESS. Among antitubercular drugs, which were the culprit drugs in 12 DRESS cases, the drug label 
for only ethambutol mentioned its potential to cause DRESS syndrome. The potential risk for the development of DRESS was not specified in the leaflets of other anti-tuberculosis drugs, such as rifampin, isoniazid, and pyrazinamide.

\section{Mortality review}

SCAR-related deaths were reported in $2.6 \%$ SCAR ICSRs (20 out of 755 SCAR ICSRs) (Table 3). With regard to DRESS syndrome, the drug-associated mortality rate was $1.2 \%$, with voriconazole, phenytoin, and oxcarbazepine each accounting for

Table 2. Common SCAR-Causing Drugs and Drug Labeling Information

\begin{tabular}{|c|c|c|c|c|c|}
\hline SCARs & $n$ & DRESS syndrome & $\mathbf{n}$ & SJS/TEN & $\mathbf{n}$ \\
\hline Total & 755 & Total & 247 & Total & 508 \\
\hline Allopurinol & 80 & Allopurinol & 28 & Allopurinol & 52 \\
\hline Carbamazepine & 65 & Carbamazepine & 24 & Carbamazepine & 41 \\
\hline Lamotrigine & 33 & Dapsone* & 9 & Lamotrigine & 25 \\
\hline Methazolamide & 16 & Lamotrigine* & 8 & Methazolamide & 16 \\
\hline Valproic acid & 16 & Oxcarbazepine & 8 & Valproic acid & 10 \\
\hline Vancomycin & 12 & Vancomycin & 7 & Paracetamol & 9 \\
\hline Dapsone & 11 & Piperacillin and enzyme inhibitor* & 7 & Amoxicillin and enzyme inhibitor & 8 \\
\hline Paracetamol & 10 & Cefotaxime* $^{*}$ & 6 & TMP/SMX & 7 \\
\hline Amoxicillin and enzyme inhibitor & 9 & Valproic acid & 6 & Ibuprofen & 6 \\
\hline Piperacillin and enzyme inhibitor & 9 & Ciprofloxacin* & 4 & Ceftriaxone & 5 \\
\hline TMP/SMX & 9 & Phenytoin* & 4 & Iloprost* & 5 \\
\hline Oxcarbazepine & 8 & Rifampicin* & 3 & Vancomycin & 5 \\
\hline Phenytoin & 8 & Others & 56 & Acetylsalicylic acid & 4 \\
\hline Ceftriaxone & 7 & Undetermined & 77 & Celecoxib & 4 \\
\hline Ibuprofen & 7 & & & Doxycycline* & 4 \\
\hline Cefotaxime & 6 & & & Mefenamic acid & 4 \\
\hline Ciprofloxacin & 6 & & & Phenytoin & 4 \\
\hline Acetylsalicylic acid & 5 & & & Amoxicillin and enzyme inhibitor & 3 \\
\hline Iloprost & 5 & & & Clarithromycin & 3 \\
\hline Celecoxib & 4 & & & Diclofenac & 3 \\
\hline Diclofenac & 4 & & & Dorzolamide & 3 \\
\hline Doxycycline & 4 & & & Enalapril & 3 \\
\hline Mefenamic acid & 4 & & & Gabapentin & 3 \\
\hline Propionic acid derivatives & 4 & & & Methotrexate & 3 \\
\hline Sulfasalazine & 4 & & & Propionic acid derivatives & 3 \\
\hline Clarithromycin & 3 & & & Tramadol & 3 \\
\hline Dorzolamide & 3 & & & Zonisamide & 3 \\
\hline Enalapril & 3 & & & Others & 105 \\
\hline Fenofibrate & 3 & & & Undetermined & 164 \\
\hline Gabapentin & 3 & & & & \\
\hline Levetiracetam & 3 & & & & \\
\hline Methotrexate & 3 & & & & \\
\hline Moxifloxacin & 3 & & & & \\
\hline Rifampicin & 3 & & & & \\
\hline Rivaroxaban & 3 & & & & \\
\hline Sunitinib & 3 & & & & \\
\hline Topiramate & 3 & & & & \\
\hline Tramadol & 3 & & & & \\
\hline Zonisamide & 3 & & & & \\
\hline Others & 127 & & & & \\
\hline Undetermined & 241 & & & & \\
\hline
\end{tabular}

SCARs, severe cutaneous adverse reactions; DRESS syndrome, drug reactions with eosinophilia and systemic symptoms syndrome; SJS/TEN, Stevens-Johnsons syndrome/Toxic epidermal necrolysis.

Others: drugs for which less than three cases were reported. Undetermined: cases in which two or more drugs were simultaneously reported as a causative agent so that the culprit drug could not be identified. TMP/SMX: trimethoprim and sulfamethoxazole.

*Drugs for which the labeling information did not include the risk of SCARs. 

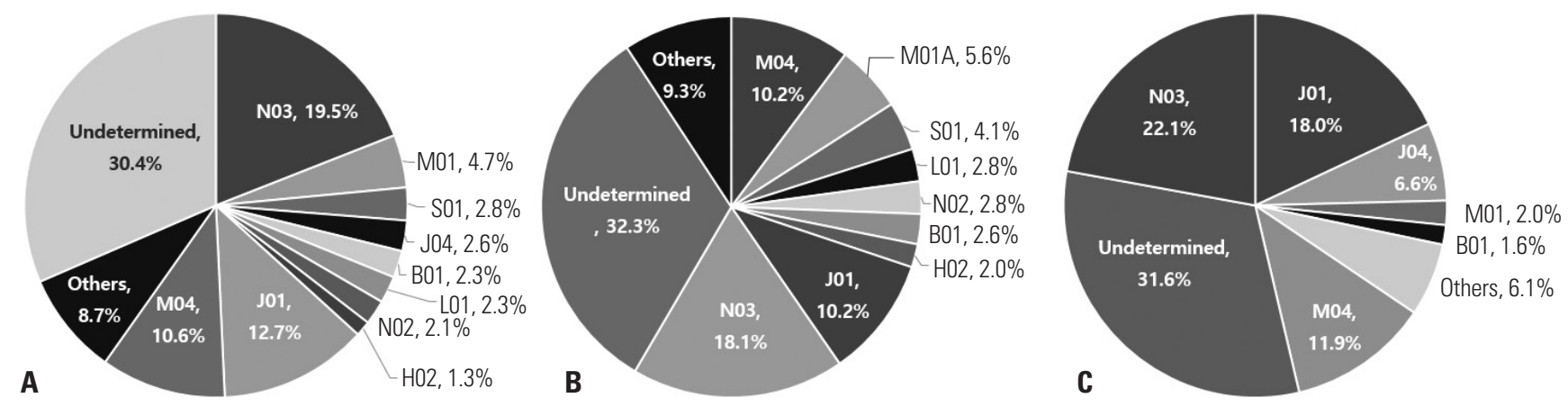

Fig. 3. Proportion of causative drugs for SCARs categorized by the main therapeutic group (second level) per ATC classification. (A) Total, (B) SJS/TEN, (C) DRESS syndrome. SCARs, severe cutaneous adverse reactions; ATC, anatomical therapeutic chemical; SJS, Stevens-Johnson syndrome; TEN, toxic epidermal necrolysis; DRESS syndrome, drug reaction with eosinophilia and systemic symptom syndrome. ATC code: B01 (antithrombotic agents), H02 (corticosteroids for systemic use), J01 (antibacterials for systemic use), J04 (antimycobacterials), L01 (antineoplastic agents), M01 (anti-inflammatory and antirheumatic products), M04 (anti-gout preparations), N02 (analgesics), N03 (antiepileptics), S01 (ophthalmologicals), and undetermined (cases in which two or more drugs were simultaneously reported as a causative agent so that the culprit drug could not be identified).

one death. The mortality rate in the SJS/TEN group was 3.3\%, with two cases attributable to allopurinol, eight to anti-infectives, two to antiepileptics, two to antitubercular drugs, and one case each to fenofibrate, methazolamide, and anti-leukemia drugs. Among the cases in which death occurred, death in two DRESS syndrome cases (voriconazole and phenytoin) and three SJS/TEN cases (cefazedone, doxycycline, and fenofibrate) was attributable to drugs whose potential to cause SCARs was not indicated on the drug label.

\section{DISCUSSION}

In this study, we investigated SCAR ICSRs submitted to the KAERS since 1989. Recently, the tendency for SCAR ICSRs has increased, with more than 100 SCAR ICSRs spontaneously submitted annually. Allopurinol and carbamazepine were the most common SCAR-causing drugs. Antiepileptics and antibacterials were the most common SCAR-inducing drug groups. The mortality rates in SCAR ICSRs in Korea were considerably lower (2.6\%) than those reported in other countries. The potential risk for SCAR development was not sufficiently specified in the drug labeling information, especially for DRESS syndrome (82.5\% of drugs in the DRESS group and $17.5 \%$ of drugs in the SJS/TEN group).

Since SCARs are rare, their epidemiology has not been sufficiently studied in Korea. Few studies have been published on the epidemiologic nature and causative drugs of SCARs in Korea. Furthermore, these studies were conducted in a single hospital or a limited number of hospitals, making it difficult to draw nationwide assumptions regarding SCAR development. Recently, a multicenter study based on the Korean Pharmacovigilance Research Network database reported that there were 100 SCAR cases from July 2009 to December $2010 .{ }^{8}$ Yang, et al. ${ }^{9}$ evaluated the nationwide claims database in Korea and reported that the incidence of SJS ranged from 3.96 to 5.03 per million and that of TEN ranged from 0.94 to 1.45 per million
Table 3. Summary of Cases of SCAR-Related Deaths and Drug Labeling Information for SCARs

\begin{tabular}{|c|c|c|c|}
\hline Diagnosis & Causative drug & ATC code & Number \\
\hline \multirow{3}{*}{ DRESS syndrome } & Voriconazole* & $J$ & 1 \\
\hline & Oxcarbazepine & $\mathrm{N}$ & 1 \\
\hline & Phenytoin* & $\mathrm{N}$ & 1 \\
\hline \multirow{15}{*}{ SJS/TEN } & Fenofibrate* & C & 1 \\
\hline & Cefazedone* & $J$ & 1 \\
\hline & $\begin{array}{l}\text { Cefoperazone/cefuroxime/ } \\
\text { amikacin }\end{array}$ & J & 1 \\
\hline & Doxycycline* & J & 1 \\
\hline & Isoniazid & J & 1 \\
\hline & $\begin{array}{l}\text { Levofloxacin/ethambutol/ } \\
\text { rifampicin/isoniazid }\end{array}$ & J & 1 \\
\hline & Levofloxacin/meropenem & J & 1 \\
\hline & Tigecycline & J & 2 \\
\hline & $\begin{array}{l}\text { Vancomycin/teicoplanin/ } \\
\text { ceftriaxone }\end{array}$ & J & 1 \\
\hline & Methotrexate & L & 1 \\
\hline & $\begin{array}{l}\text { Vincristine/rituximab/ } \\
\text { prednisolone/doxorubicin/ } \\
\text { cyclophosphamide }\end{array}$ & L & 1 \\
\hline & Allopurinol & M & 2 \\
\hline & Carbamazepine & $\mathrm{N}$ & 1 \\
\hline & Valproic acid & $\mathrm{N}$ & 1 \\
\hline & Methazolamide & S & 1 \\
\hline
\end{tabular}

SCARs, severe cutaneous adverse reactions; DRESS syndrome, drug reactions with eosinophilia and systemic symptom syndrome; SJS/TEN, StevensJohnsons syndrome/toxic epidermal necrolysis; ATC, anatomical therapeutic chemical.

*Drugs for which the labeling information did not include the risk of SCARs.

from 2010 to 2013. In our study, 755 SCAR cases were reported since ADE spontaneous reporting began in Korea. The number of ICSRs of SCARs has been increasing in Korea. More than 100 SCAR cases have been spontaneously reported every year since 2010. A total of 193 cases was spontaneously re- 
ported to KAERS in 2013. It remains unclear whether the increasing trend of SCAR ICSRs resulted from an actual increase in the development of SCARs in Korea. Although SCAR ICSRs are steadily increasing, the proportion of SCAR ICSRs among ICSRs of overall adverse events is declining. Thus, the increasing SCAR ICSRs seem to be the result of active pharmacovigilance by regional pharmacovigilance centers rather than being truly an increase in SCAR ICSRs.

Although allopurinol-induced and carbamazepine-induced SCARs are both common in Korea and in other countries, ${ }^{10}$ the epidemiology of SCARs in Korea might be somewhat different from that in other countries. ${ }^{11}$ Allopurinol-induced SCARs are common in Korea and Taiwan. The $H L A-B * 58: 01$ genotype is a well-known risk factor for allopurinol-induced SCARs in the Han Chinese in Taiwan (OR 580.3). ${ }^{12}$ The allele frequency of $H L A-B^{*} 58: 01$ is $12.2 \%$ in Koreans ${ }^{13}$ and $9-11 \%$ in the Han Chinese, ${ }^{12}$ such that $H L A-B * 58: 01$ screening proved to be effective to prevent allopurinol-induced SCARs in Korea ${ }^{14}$ and Taiwan. ${ }^{15}$ However, the allele frequency of $H L A-B * 58: 01$ is lower in Japanese (1-2\%) ${ }^{16}$ and Caucasians (1-6\%). ${ }^{17}$ Carbamazepine-induced SCAR is also common in Korea and Taiwan. In the Han Chinese, the incidence of carbamazepine-induced SCARs was more than 10-fold higher than that in other ethnic groups. ${ }^{18}$ The $H L A-B^{*} 15: 02$ haplotype is clearly associated with carbamazepine-induced SCARs (OR 2504.0).${ }^{19}$ However, the $H L A-B 15: 02$ allele frequency was show to be quite low $(0.41 \%)$ in the Korean population, compared to that in Han Chinese (10-15\%). ${ }^{19,20}$ Instead, HLA-A3101 is highly associated with carbamazepine-induced SJS in Korean population. ${ }^{6}$ Abacavir, which is an anti-retroviral drug used to treat HIV/AIDS, is known to frequently elicit hypersensitivity reactions in individuals of European descent. ${ }^{21}$ However, abacavir-induced SCAR ICSRs have not been reported in Korea. Methazolamide was one of the common SCAR-causing drugs in our study. HLA$B^{*}$ 59:01 is a known risk factor for methazolamide-induced SCARs. The $H L A-B^{*} 59: 01$ allele is present in $1-2 \%$ of Korean population, but in $<0.5 \%$ of Chinese population, and is extremely rare in Caucasian individuals. ${ }^{22}$ To date, methazolamide-related SCARs have been reported only in Koreans, Japanese, and Chinese.

The mortality rate in SCAR ICSRs in our study was much lower than rates in other studies. ${ }^{21,23-25}$ Mortality due to SJS and TEN has been previously reported to be $1-13 \%$ and $30-50 \%$, respectively. In our study, the mortality rates in SJS ICSRs and TEN ICSRs were $2.9 \%$ ( 12 cases among 408 SJS ICSRs) and 5\% (5 cases among 100 TEN ISCRs), respectively. The mortality rates in DRESS ICSRs were also quite low in our study (1.2\%), compared to those in other studies (approximately 10\%). ${ }^{26,27} \mathrm{It}$ is difficult to generalize whether the mortality rate associated with SCARs in Korea is lower than rates in other countries. We retrospectively reviewed the SCAR ICSRs in the KIDS-KD. Sekula, et al. ${ }^{24}$ reported that a considerable number of SCARrelated deaths occurred after successful treatment and follow- ing hospital discharge. If the outcomes in SCAR ICSRs are not properly followed up, SCAR-related mortality might be underestimated. However, Yang, et al. ${ }^{9}$ analyzed Korean national health insurance data and reported that the mortality associated with TEN was approximately $15 \%$, which was still lower than that in other countries.

The labeling information of many drugs in our study did not contain relevant warnings for the possibility of SCAR development. Information about the potential risk for DRESS syndrome was not mentioned in the labeling information for approximately two-thirds of the DRESS-causing drugs in our study. Although approximately $5 \%$ of all DRESS syndrome cases were attributable to dapsone and lamotrigine, the relevant labeling information for DRESS syndrome was not included for either drug. Among the 10 common DRESS syndrome-inducing drugs, the risk for DRESS syndrome was mentioned in the labeling information for only five drugs. Considering the severity of SCARs, it will be necessary to mention the potential risk of SCAR development in the labeling information during post-marketing surveillance.

This study has some limitations. First, it was based on the ICSRs from the KIDS-KD, which is based on a spontaneous $\mathrm{ADE}$ reporting system. Therefore, the numbers may be underestimated due to underreporting. Second, the information in the ICSRs might not be sufficient to fully evaluate the complex epidemiologic nature of SCARs. Important epidemiologic factors were sometimes omitted in the ICSRs. Notably, multiple drugs taken at the time of symptom onset were registered as causative agents in cases in which the SCAR-inducing drug could not be determined. To overcome these limitations, the SCAR Special Interest Group was formed and a web-based SCAR registry was established in Korea. The SCAR registry enrolled patients with SCARs and gathered detailed epidemiologic information on baseline characteristics and various SCAR-associated phenotypes, in addition to collecting blood samples for the evaluation of genetic factors, such as $H L A$ haplotype.

We investigated the epidemiologic characteristics and causative drugs of SCARs in Korea using a nationwide ICSR database. Allopurinol was the most common SCAR-inducing individual drug in both the DRESS and SJS/TEN groups. Antiepileptics were the most common SCAR-causing drug group, followed by antibacterials and NSAIDs. We found that, despite the seriousness of SCARs, the potential of a drug to cause SCARs was not mentioned in the labeling information for more than half of the culprit agents studied.

\section{ACKNOWLEDGEMENTS}

This research was supported by a grant from the Ministry of Food and Drug Safety to the regional pharmacovigilance center in 2017. 


\section{AUTHOR CONTRIBUTIONS}

Conceptualization: Min-Gyu Kang, Han-Ki Park, Hye-Ryun Kang. Data curation: Min-Gyu Kang, Han-Ki Park. Formal analysis: MinGyu Kang, Han-Ki Park. Funding acquisition: Min-Gyu Kang, HyeRyun Kang. Investigation: Min-Gyu Kang, Hani-Ki Park, Hye-Ryun Kang. Methodology: Min-Gyu Kang, Dong-Yoon Kang, Min-Seok Yang, Kyung-Hee Sohn. Project administration: Min-Gyu Kang. Resources: Min-Gyu Kang, Han-Ki Park. Software: Min-Gyu Kang. Supervision: Min-Seok Yang, Hye-Ryun Kang, Ju-Yeun Lee. Validation: Min-Gyu Kang, Kyung-Hee Sohn, Ju-Yeun Lee. Visualization: MinGyu Kang. Writing_original draft: Min-Gyu Kang, Hye-Ryun Kang. Writing - review \& editing: Min-Gyu Kang, Dong-Yoon Kang, MinSeok Yang, Ju-Yeun Lee, Han-Ki Park, Kyung-Hee Sohn, Hye-Ryun Kang.

\section{ORCID iDs}

Min-Gyu Kang

Kyung-Hee Sohn

Dong-Yoon Kang

Han-Ki Park

Min-Suk Yang

Ju-Yeun Lee

Hye-Ryun Kang https://orcid.org/0000-0002-3239-4492 https://orcid.org/0000-0001-8407-8080 https://orcid.org/0000-0003-4283-2633 https://orcid.org/0000-0002-5460-9917 https://orcid.org/0000-0002-9861-0530 https://orcid.org/0000-0002-2261-7330 https://orcid.org/0000-0002-2317-4201

\section{REFERENCES}

1. International drug monitoring: the role of national centres. Report of a WHO meeting. World Health Organ Tech Rep Ser 1972;498:1-25.

2. Lazarou J, Pomeranz BH, Corey PN. Incidence of adverse drug reactions in hospitalized patients: a meta-analysis of prospective studies. JAMA 1998;279:1200-5.

3. GBD 2013 Mortality and Causes of Death Collaborators. Global, regional, and national age-sex specific all-cause and cause-specific mortality for 240 causes of death, 1990-2013: a systematic analysis for the Global Burden of Disease Study 2013. Lancet 2015;385:117-71.

4. Swanson L, Colven RM. Approach to the patient with a suspected cutaneous adverse drug reaction. Med Clin North Am 2015;99: 1337-48.

5. Yang MS, Kang MG, Jung JW, Song WJ, Kang HR, Cho SH, et al. Clinical features and prognostic factors in severe cutaneous drug reactions. Int Arch Allergy Immunol 2013;162:346-54.

6. Kim SH, Lee KW, Song WJ, Kim SH, Jee YK, Lee SM, et al. Carbamazepine-induced severe cutaneous adverse reactions and HLA genotypes in Koreans. Epilepsy Res 2011;97:190-7.

7. Kang HR, Jee YK, Kim YS, Lee CH, Jung JW, Kim SH, et al. Positive and negative associations of HLA class I alleles with allopurinolinduced SCARs in Koreans. Pharmacogenet Genomics 2011;21: 303-7.

8. Kim MY, Yang MS, Kang HR, Cho SH, Min KU. Analysis of drugs causing severe cutaneous adverse reactions, based on the Korean Database of spontaneously reported adverse drug reactions. Korean J Med 2014;86:710-21.

9. Yang MS, Lee JY, Kim J, Kim GW, Kim BK, Kim JY, et al. Incidence of Stevens-Johnson Syndrome and toxic epidermal necrolysis: a nationwide population-based study using National Health Insurance Database in Korea. PLoS One 2016;11:e0165933.

10. Park HJ, Kim YJ, Kim DH, Kim J, Park KH, Park JW, et al. HLA allele frequencies in 5802 Koreans: varied allele types associated with SJS/TEN according to culprit drugs. Yonsei Med J 2016;57: 118-26.

11. Jung JW, Kim JY, Park IW, Choi BW, Kang HR. Genetic markers of severe cutaneous adverse reactions. Korean J Intern Med 2018;33: 867-75.

12. Hung SI, Chung WH, Liou LB, Chu CC, Lin M, Huang HP, et al. HLA-B*5801 allele as a genetic marker for severe cutaneous adverse reactions caused by allopurinol. Proc Natl Acad Sci U S A 2005; 102:4134-9.

13. Lee KW, Oh DH, Lee $C$, Yang SY. Allelic and haplotypic diversity of HLA-A, -B, -C, -DRB1, and -DQB1 genes in the Korean population. Tissue Antigens 2005;65:437-47.

14. Jung JW, Kim DK, Park HW, Oh KH, Joo KW, Kim YS, et al. An effective strategy to prevent allopurinol-induced hypersensitivity by HLA typing. Genet Med 2015;17:807-14.

15. Ko TM, Tsai CY, Chen SY, Chen KS, Yu KH, Chu CS, et al. Use of HLA-B*58:01 genotyping to prevent allopurinol induced severe cutaneous adverse reactions in Taiwan: national prospective cohort study. BMJ 2015;351:h4848.

16. Niihara H, Kaneko S, Ito T, Sugamori T, Takahashi N, Kohno K, et al. HLA-B*58:01 strongly associates with allopurinol-induced adverse drug reactions in a Japanese sample population. J Dermatol Sci 2013;71:150-2.

17. Lonjou C, Borot N, Sekula P, Ledger N, Thomas L, Halevy S, et al. A European study of HLA-B in Stevens-Johnson syndrome and toxic epidermal necrolysis related to five high-risk drugs. Pharmacogenet Genomics 2008;18:99-107.

18. Chen P, Lin JJ, Lu CS, Ong CT, Hsieh PF, Yang CC, et al. Carbamazepine-induced toxic effects and HLA-B*1502 screening in Taiwan. N Engl J Med 2011;364:1126-33.

19. Hung SI, Chung WH, Jee SH, Chen WC, Chang YT, Lee WR, et al. Genetic susceptibility to carbamazepine-induced cutaneous adverse drug reactions. Pharmacogenet Genomics 2006;16:297-306.

20. Lee KW, Jeon H, Park JY. HLA-B* 15 diversity in the Korean population. Tissue Antigens 2000;56:428-35.

21. Mockenhaupt M, Viboud C, Dunant A, Naldi L, Halevy S, Bouwes Bavinck JN, et al. Stevens-Johnson syndrome and toxic epidermal necrolysis: assessment of medication risks with emphasis on recently marketed drugs. The EuroSCAR-study. J Invest Dermatol 2008;128:35-44.

22. Shu C, Shu D, Tie D, Yu M, Zhang R, Wang T, et al. Toxic epidermal necrolysis induced by methazolamide in a Chinese-Korean man carrying HLA-B*59:01. Int J Dermatol 2015;54:1242-5.

23. Schöpf E, Stühmer A, Rzany B, Victor N, Zentgraf R, Kapp JF. Toxic epidermal necrolysis and Stevens-Johnson syndrome. An epidemiologic study from West Germany. Arch Dermatol 1991;127: 839-42.

24. Sekula P, Dunant A, Mockenhaupt M, Naldi L, Bouwes Bavinck JN, Halevy S, et al. Comprehensive survival analysis of a cohort of patients with Stevens-Johnson syndrome and toxic epidermal necrolysis. J Invest Dermatol 2013;133:1197-204.

25. Sekula P, Liss Y, Davidovici B, Dunant A, Roujeau JC, Kardaun S, et al. Evaluation of SCORTEN on a cohort of patients with StevensJohnson syndrome and toxic epidermal necrolysis included in the RegiSCAR study. J Burn Care Res 2011;32:237-45.

26. Eshki M, Allanore L, Musette P, Milpied B, Grange A, Guillaume JC, et al. Twelve-year analysis of severe cases of drug reaction with eosinophilia and systemic symptoms: a cause of unpredictable multiorgan failure. Arch Dermatol 2009;145:67-72.

27. Chiou CC, Yang LC, Hung SI, Chang YC, Kuo TT, Ho HC, et al. Clinicopathological features and prognosis of drug rash with eosinophilia and systemic symptoms: a study of 30 cases in Taiwan. J Eur Acad Dermatol Venereol 2008;22:1044-9. 